\title{
Criminologie
}

\section{Sommes-nous tous des assassins?}

\section{Alice Parizeau}

Volume 8, numéro 1-2, 1975

Délinquance juvénile au Québec

URI : https://id.erudit.org/iderudit/017044ar

DOI : https://doi.org/10.7202/017044ar

Aller au sommaire du numéro

Éditeur(s)

Les Presses de l'Université de Montréal

ISSN

0316-0041 (imprimé)

1492-1367 (numérique)

Découvrir la revue

Citer cet article

Parizeau, A. (1975). Sommes-nous tous des assassins ? Criminologie, 8(1-2), 167-174. https://doi.org/10.7202/017044ar d'utilisation que vous pouvez consulter en ligne.

https://apropos.erudit.org/fr/usagers/politique-dutilisation/ 
SOMMES-NOUS TOUS DES ASSASSINS?

Alice Parizeau 
C'est le 21 octobre dernier qu'a eu lieu le colloque organisé par la Société de criminologie de Montréal, qui portait, comme l'annonçait la lettre d'invitation, sur le problème « des enfants battus, sujets de mauvais traitements physiques de la part de leurs parents ».

Lors de cette réunion au pavillon des Sciences sociales de l'Université de Montréal, le docteur Bernard Méthot, de l'hôpital Sainte-Justine, et le docteur Jean-Paul Valcourt, de l'Institut de médecine légale, ont présenté deux documentaires. L'un était en couleur, l'autre en noir et blanc. Ils montraient les corps des enfants blessés, mutilés, lacérés au couteau, étranglés, morts par asphyxie à la suite d'immersion prolongée dans une baignoire...

Sur des brancards, sagement alignés, munis de numéros d'immatriculation, défilaient les cadavres de bébés et d'enfants martyres. Les médecins expliquaient, dans leurs commentaires, les situations dans lesquelles ils sont devenus des victimes, soit de parents, soit de parents nourriciers, c'est-à-dire de gens auxquels la société, par l'entremise d'un juge des enfants, a confié leur sort, moyennant une certaine rémunération...

On a discuté ensuite, lors des débats, des couples désunis, des mères célibataires, des milieux désavantagés, de la pilule et des problèmes que doit affronter la famille urbaine. Les médecins, pour leur part, ont expliqué les difficultés de faire la preuve devant la cour du Bien-Etre social, tandis que le ministre de la Justice, qui était présent en tant qu'invité, ne pouvait pas demander à son collègue du ministère des Affaires sociales pourquoi 
la prévention n'existe pas et pourquoi les hôpitaux ne disposent pas des services sociaux adéquats.

Est-il possible de les organiser?

Selon la bonne vieille tradition de notre société, l'autorité parentale demeure un dogme. La société se protège en versant aux parents une aide sociale, s'ils sont incapables de gagner leur vie, libre à eux de s'occuper de leurs enfants. De la même manière, si on nous permet cette comparaison, la société se protège contre les animaux qui peuvent devenir dangereux en versant à la Société protectrice des animaux des allocations l'aidant à s'occuper des chiens perdus sans collier...

En somme, bien que les enfants demeurent la plus grande richesse de toute collectivité, bien qu'ils sont la seule garantie de sa survie, on prend pour acquis qu'on ne peut les aider qu'à travers les parents, ou encore un foyer de substitution, en attendant qu'ils grandissent et deviennent d'âge à être jugés, selon la loi, comme des jeunes délinquants...

Car toutes les recherches le prouvent : la grande majorité des enfants et adolescents entendus devant les tribunaux sont des mal-aimés, sinon des victimes de l'indifférence, de la bêtise, des mauvais traitements et de la cruauté des adultes.

A leur égard, cependant, la permissivité de la collectivité demeure entière. Le code criminel prévoit une peine de $\$ 500$, ou encore la détention dont la durée maximum ne peut dépasser deux ans.

Une petite fille, qui avoue en toute candeur qu'elle est plus heureuse dans une institution que chez elle, car * là, au moins, elle peut dormir tranquille ", tandis que chez elle son père se livre à des pratiques incestueuses, risque de se faire considérer comme un enfant « vicieux », surtout si le père gagne sa vie et mène en apparence une existence dite normale. Pour faire la preuve, il faut un constat de visu...

\section{L'enfant est sans défense !}

Les garçons et les filles battus et maltraités, sont parfois soignés par des médecins ; mais règle générale, ils se taisent parce qu'ils ont trop peur de parler, sachant ce qui les attend une fois de retour à la maison. 
Les enfants assassinés sauvagement par des parents, ou par des parents nourriciers, ne suscitent qu'un sentiment d'horreur fort passager. En pratique, devant les tribunaux, l'avocat plaidera l'irresponsabilité de la mère ou du père, pour cause de maladie mentale, et il va gagner.

La société, nous tous collectivement, nous nous répétons 《il s'agit de cas extrêmes, d'une infime minorité », et cela suffit pour nous donner bonne conscience. Il y a là un illogisme que les collectivités nourrissent à dessein. Les adultes votent, les enfants de moins de dix-huit ans ne votent pas. Il convient donc surtout de défendre les droits des adultes, puisque cela est rentable électoralement. Dès lors, la prévention demeure inexistante ou inefficace. On prend pour acquis que seules les femmes qui ne désirent pas avoir un enfant peuvent être cruelles et on distribue généreusement la pilule.

Lors du colloque, cependant, le docteur Méthot a affirmé à deux reprises que parmi les enfants martyrs qu'il a examinés à Sainte-Justine, il y avait surtout des enfants désirés...

La pilule ne semble pas, en somme, être un médicament universel contre la cruauté, l'indifférence et la bêtise.

La prévention n'est-ce pas, par conséquent, la nécessité absolue de vérifications préventives et le rejet de dogmes et tabous selon lesquels le fait d'enfanter suscite automatiquement un amour parental sans faille?

Mais voilà un domaine où, en vertu de notre hypocrisie collective, on se plaît à défendre les droits à la liberté individuelle!

Quand les cours supérieures statuent qu'on peut perquisitionner chez vous sans mandat, quand on décide du jour au lendemain d'arrêter un millier de personnes sous prétexte qu'éventuellement elles pourraient être dangereuses, comme ça, sans aucune preuve, ni même présomption "probable et raisonnable ", cela est considéré comme normal. Notre société semble aimer passionnément "être protégée ». Mais quand des milliers d'enfants sont maltraités physiquement, moralement ou mentalement, chaque jour, dans nos grandes villes, on prétend que les vérifications seraient inacceptables, puisqu'il y a nécessité absolue de protéger les libertés individuelles des parents qui sont des électeurs en puissance. 
Certes, des infirmières visiteuses et des travailleurs sociaux qui ne se rendent que dans les quartiers désavantagés, c'est du paternalisme, mais on comprend mal pourquoi notre collectivité ne pourrait pas accepter que de telles visites puissent avoir lieu régulièrement et systématiquement dans tous les foyers où un enfant vient de naître. L'enregistrement des naissances se fait automatiquement dans les hôpitaux, il suffirait donc de disposer des services sociaux, suffisamment importants numériquement, rattachés aux hôpitaux, pour qu'on puisse effectuer des contrôles élémentaires, annuels, mensuels, ou hebdomadaires, selon les besoins de chaque famille.

On peut se demander aussi comment se fait-il que les médecins qui assistent les femmes avant et lors de l'accouchement ne se rendent pas compte de leur état mental et ne signalent pas le cas aux services sociaux immédiatement, au lieu d'attendre qu'on leur emmène un autre bébé, ou enfant, au corps lacéré de coups de couteau. Jusqu'où faut-il aller dans l'horreur pour qu'on accepte enfin de telles mesures préventives élémentaires qui d'ailleurs existent depuis des années dans la vieille Europe?

Au Québec, autrefois, le clergé assumait cette mission, désormais il perd prise sur le milieu et doit être aidé, ou encore remplacé par des services sociaux. Cela coûte cher, on le sait, mais les frais en termes de salaires seront certainement moins élevés que ceux qu'il faudrait assumer pour enlever l'enfant à la famille et le placer dans une institution.

Si l'on accepte, par ailleurs, de dépenser des sommes de plus en plus astronomiques pour assumer à la collectivité la protection de la police, comment se fait-il qu'on soit aussi avare de nos deniers quand il s'agit de protéger des enfants?

Des travaux et des recherches prouvent que les enfants ne naissent pas criminels, mais qu'ils le deviennent avec l'aide, généralement inconsciente, Dieu merci, de leurs parents. Parce qu'on ne peut choisir sa famille, on finit, en somme par avoir des comportements marginaux et on défile tôt ou tard devant monsieur le juge...

Dans les articles que contient cette livraison de la revue, les auteurs ont traité des aspects légaux de la protection de l'enfance, de la définition de ce que signifie en fait le concept de a délinquance juvénile » et Marcel Fréchette a expliqué à ce propos que 
ce concept ne s'applique pleinement qu'à une minorité. La majorité, et même selon toute probabilité, cette minorité également, ce ne sont que des victimes des adultes qui tout en n'étant pas des « enfants battus, sujets de mauvais traitements », qu'on peut montrer morts sur un brancard, ont été tués moralement et psychiquement.

Il est permis de conclure, en somme, que la collectivité se doit de prendre en charge la défense de l'enfance malheureuse avant qu'elle ne devienne délinquante.

Cela est-il possible?

Nous nous permettons de répondre oui, en nous basant sur certaines statistiques.

C'est ainsi que les relevés que nous avons effectués à la cour du Bien Etre social de Montréal, en 1968, pour le compte de la Commission Prévost, nous ont permis de constater que pour plus de 7000 jeunes qui ont passé devant cette cour, il n'y avait que quelques dizaines de juifs et quelques centaines de protestants canadiens-anglais. Ces pourcentages, il va sans dire, ne correspondent nullement aux rapports numériques entre les ethnies, ou groupes d'appartenance religieuse, concernées. Parallèlement il n'est pas vrai que la délinquance juvénile n'existe pas dans ces groupes, même si, toute proportion gardée, leur statut économique est supérieur à celui de certains milieux désavantagés francophones. Le plus élémentaire raisonnement logique permet de constater, sur cette base, que la communauté juive, ainsi que la communauté canadienne-anglaise, parviennent à réunir des fonds privés, tout en ayant comme tout le monde des fonds publics, et à mieux contrôler ensuite les problèmes familiaux, même s'il faut pour cela enfreindre, d'une certaine façon, la fameuse règle de l'autorité parentale.

Lors des recherches que nous avons effectuées dans les milieux anglophones de Pointe-Claire nous avons pu confirmer cette hypothèse. On y a organisé entre autres, avec, à l'origine, un groupe d'une centaine de bénévoles, un service téléphonique et un service d'hébergement temporaire des enfants qui demandent de l'aide, ou qui ont été dépistés par des travailleurs de rue comme devant être assistés...

Quand admettra-t-on enfin dans notre milieu que la protection de l'enfance est un problème trop sérieux pour qu'on le confie 
au « pouvoir * et que nous nous devons de mettre en place des structures communautaires, de quartiers et de régions, puis, en deuxième temps seulement, exiger des fonds pour les standardiser et les institutionnaliser?

Après tout, les précédents historiques de ce type d'action ne manquent pas. Tout le système de libération, de probation et de réinsection sociale a été organisé ainsi avant d'être pris en charge par l'État. Et il ne s'agissait là, après tout, que d'adultes...

En attendant, en regardant les documentaires présentés par les médecins au colloque du 21 octobre, on ne pouvait que se poser cette simple question : Sommes-nous tous, vraiment, des assassins? 